\title{
Investigation of Factors Considered by Health Technology Assessment Agencies in Eight Countries
}

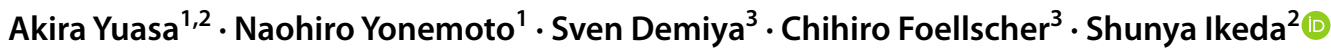

Accepted: 3 October 2020 / Published online: 27 October 2020

(c) The Author(s) 2020

\begin{abstract}
Background Health technology assessment (HTA) organizations play a crucial role in optimizing healthcare resources, but the factors influencing decision making vary by country.

Objective HTAs of cancer and hepatitis C drugs were evaluated across developed countries to understand differences in decision processes and criteria.

Methods The HTA organizations evaluated are from France, Germany, Italy, Spain, the United Kingdom (UK), Australia, Canada and Japan. Economic evaluation types and 28 factors in the following categories were evaluated: clinical uncertainties/issues; disease/population/treatment consideration factors including National Institute for Health and Care Excellence's (NICE) special circumstances factors (e.g. end-of-life and innovation); and International Society for Pharmacoeconomics and Outcomes Research (ISPOR) additional value elements. Qualitative and correspondence analyses were conducted to assess the differences across organizations.

Results Incremental cost-effectiveness ratio (ICER) using quality-adjusted life-year (QALY) was evaluated in Canada, the UK, Australia and Japan. The highest observed clinical uncertainties were clinical benefits and comparator. For cancer drugs, correspondence analysis showed France, Australia, Canada and the UK to have common attributes observed, such as unmet needs and stakeholder persuasion. In addition, the UK reported end-of-life, issues around current treatment and innovation, whereas Germany reported manageable/insignificant adverse events more frequently. Finally, fear of contagion, equity and scientific spillover value elements were only observed in Australia.

Conclusion Although clinical factors play a predominant role in the decision to reimburse medicine, HTA organizations consider additional aspects as well. If the methodology of HTA was clearly outlined, there would be more transparency in HTA systems leading to better understanding amongst stakeholders about decision making.
\end{abstract}

Electronic supplementary material The online version of this article (https://doi.org/10.1007/s41669-020-00235-6) contains supplementary material, which is available to authorized users.

Shunya Ikeda

shunya@iuhw.ac.jp

Pfizer Japan Inc, Tokyo, Japan

2 Graduate School of Medicine, International University of Health and Welfare, 1-26, Akasaka 4-chome, Minato-ku, Tokyo 107-8402, Japan

3 IQVIA Solutions Japan K.K., Tokyo, Japan

\section{Key Points for Decision Makers}

This study gives insight into the current available information given directly in HTA dossiers that widely differs across countries and highlights the consideration of other factors beyond clinical factors.

Stakeholder persuasion and unmet needs were the relative common factors for decision making across all HTA organizations. 


\section{Introduction}

The overall expenditure for healthcare is increasing more than the total gross domestic product (GDP) of most countries globally. A report on public health spending by the World Health Organization (WHO) between 2000 and 2016 revealed an average annual rise of $6 \%$ and $4 \%$ in the total healthcare expenditure in low/middle and high-income countries, respectively [1]. The increasing economic burden of the healthcare sector can be mostly attributed to hospital care costs, physicians and clinical services and prescription drug costs [2, 3]. Cancer and hepatitis $\mathrm{C}$ are two therapeutic areas associated with the highest economic burden, as reported by the WHO [4, 5].

Health Technology Assessment (HTA) organizations can make decisions on financial implications of any intervention to the healthcare system of a country based on different economic analyses like budget impact analysis and cost-effectiveness analysis [6].

The decisions made by HTA organizations have a great impact on the therapeutic market. Based on the HTA recommendations, the patient market access to any therapeutic may increase or decrease. Pharmaceutical companies also observe the trends of HTA recommendations made in the past when designing and conducting clinical trials for drugs. This helps them in generating necessary evidence required for applying for reimbursement of their products [7].

Various studies have been conducted to explore the patterns of decision making by HTA organizations. Studies have highlighted disparities in HTA recommendations related to HTA processes, contextual aspects [8], methodological differences [9], levels of scientific evidence and patient evidence, levels of uncertainties [10] and willingness-to-pay thresholds [11]. Two studies, Dakin et al. [12] and Nicod [13], compared the characteristic elements and factors considered by HTA organizations for decision making. The assessment approach of the HTA organization of the United Kingdom (UK) - the National Institute for Health and Care Excellence (NICE) — was studied by Rawlins et al. [14]. They reported that the decisions of NICE are influenced by two types of judgements-scientific and social values. The former considers the clinical and economic evidence while the latter considers the severity of the underlying illness, end-of-life treatments, stakeholder persuasion, significant innovation, disadvantaged populations and children [14]. In recent years, the International Society for Pharmacoeconomics and Outcomes Research (ISPOR) Special Task Force has suggested the inclusion of 12 potential elements of value in healthcare for broadening the evaluation items of HTA organizations into costeffectiveness evaluation of new interventions [15].
There are only a few studies comparing the overall process of decision making in HTA organizations in developed countries, such as Japan, which introduced a pilot HTA programme in Japan in April 2016. The objective of the present study was to understand the differences in the process and factors involved in decision making for HTA organizations across eight countries-France, Germany, Italy, Spain, UK, Australia, Canada and Japan. Inclusion of the 12 potential elements of value (suggested by ISPOR) across the HTA organizations of these eight countries was also explored.

\section{Methods}

\subsection{Selection of Drugs and Health Technology Assessment (HTA) Organizations}

The targeted literature review (TLR) was focused on four target drugs for cancer (Opdivo ${ }^{\circledR}$ [nivolumab], Keytruda ${ }^{\circledR}$ [pembrolizumab], Kadcyla ${ }^{\circledR}$ [ado-trastuzumab emtansine], Ibrance ${ }^{\circledR}$ [palbociclib]) and five target drugs for hepatitis C (Daklinza ${ }^{\circledR}$ [daclatasvir], Sunvepra ${ }^{\circledR}$ [asunaprevir], Viekirax ${ }^{\circledR}$ [ombitasvir], Harvoni ${ }^{\circledR}$ [ledipasvir/sofosbuvir], Sovaldi ${ }^{\circledR}$ [sofosbuvir]) that were evaluated up until August 2019. The dossiers of these nine target drugs were compared across the following HTA organizations-Agencia de Evaluación de Tecnologías Sanitarias de Andalucía (AETSA; Spain), Agenzia Italiana del Farmaco (AIFA; Italy), Center for Outcomes Research and Economic Evaluation for Health/ Central Social Insurance Medical Council (C2H/Chuikyo; Japan), Canadian Agency for Drugs and Technologies in Health/pan-Canadian Oncology Drug Review (CADTH/ pCODR; Canada), Haute Autorité de Santé (HAS; France), Institute for Quality and Efficiency in Healthcare (IQWiG; Germany), NICE (UK) and Pharmaceutical Benefits Advisory Committee (PBAC; Australia). In Spain, there are seven autonomous regions with HTA agencies (Catalonia, Andalusia, the Basque Country, Valencia, Galicia, Navarre and the Canary Islands) that are responsible for their own healthcare systems [16]. AETSA was chosen for this review as Andalucia is the largest region in Spain with 8,405,294 inhabitants (July 2018) [17]. Descriptive information is provided in Supplemental Table S1 (see electronic supplementary material [ESM]).

Different types of assessments were included: original submission, extension of indication, resubmission and unavailable status. However, the publication status was labelled as completed for each. Cancer and hepatitis $\mathrm{C}$ drugs were selected due to their high economic burden $[3,4]$ and the aforementioned eight HTA organizations were selected due to similarities in their countries' health expenditures and GDP per capita $[18,19]$. 


\subsection{Data Collection}

The TLR was conducted from two sources-HTA accelerator (HTAA; IQVIA's HTA dossier literature platform) and the evidence or dossiers available on the homepages of HTA organizations from the respective countries $[20,21]$. The articles were selected by one researcher and cross-checked by another researcher to ensure article selection met the selection criteria.

These dossiers were evaluated for factors mentioned in Sect. 2.3. Factors found in the HTAA database were verified by a local person in charge of each country and verified through the homepage and dossiers of each target HTA organization. Additional factors were also collected from HTA dossiers by C.F., R.D.M., H.M. and R.M. (see Acknowledgements section). English versions of the dossier were used, if available, and otherwise Google translate was used to translate French, German, Italian and Spanish dossiers to English, as was done by Verghese et al. [22]. These additional factors were extracted by C.F. and H.M. and cross-checked by R.D.M and R.M. to ensure that the collected information adhered to the definitions provided in Sect. 2.3.

Each original, resubmission and extension of indication dossiers were evaluated individually. If multiple subgroups, such as cohorts or genotypes, were evaluated in one single dossier, each factor found was only counted once, meaning a factor is not necessarily reported for all listed subgroups. Furthermore, each factor was evaluated as being reported regardless of whether that factor was directly considered or involved in the decision-making process.

The details of the population/intervention/comparison/ outcome/study design (PICOS) criteria and the inclusion/ exclusion criteria for each dossier in the TLR are provided in Supplemental Table S2 and Supplemental Table S3, respectively (see ESM).

\subsection{Variables}

An initial list of 34 evaluation factors was created from studies conducted by Nicod [13], Rawlins et al. [14] and Lakdawalla et al. [15]. This list was then refined by removing duplicates and redefining some factors to arrive at our own list comprising 28 factors, which were grouped into the following four categories for data analysis: type of economic evaluation, clinical uncertainties and issues, consideration factors (consisting of disease considerations, population considerations and treatment considerations, which were identified separately) and additional elements of value (see Supplemental Table S2in the ESM for more detail). The economic evaluations were observed based on the economic analysis provided in the dossiers, such as incremental cost-effectiveness ratio (ICER) with quality-adjusted life-years (QALY), cost only or no analysis at all. The clinical factors were observed from two viewpoints-uncertainties and issues. Uncertainties are defined as unclear or insufficient clinical evidences that hindered the ability to obtain a solid judgement of the assessed technology reported by the HTA organizations. On the other hand, issues are defined as clinical factors that were expressed as being incorrect or problematic by the HTA organizations. They are identified by the exact and/or similar expressions in the dossier.

A detailed list of the 28 factors along with their definitions is provided in Supplemental Table S4 (see ESM).

\subsection{Data Analysis}

The data analysis was performed using $\mathrm{R}$ version 3.6 .0 and the graphs were produced using R package 'ggplot2'. The data collected from the HTAA database and HTA dossiers was transformed into categorical, nominal or dichotomous variables. Descriptive statistics were used to measure the frequencies of each element to compare across the eight HTA organizations. Bar charts were used in order to compare the number of cases per factor across the organizations.

For quantitatively understanding the association between the factors considered across various HTA organizations, corresponding analysis was used. In this analysis, categorical variables were explored using visual technique-based $R$ packages, namely 'FactoMineR' and 'factoextra' [23, 24]. Pearson's chi-square test was performed to determine the presence of any significant relationship between two categorical variables. In this test, if the null hypothesis was rejected, the categorical variables were assumed to be dependent while if it was not rejected, the categorical variables were assumed to be independent [25]. Eigenvalues (values corresponding to the amount of information retained by each dimension) were examined to determine the number of dimensions to finally plot the correspondence analysis, where each axis represented a dimension. The cumulative variance was obtained by adding all the eigenvalues.

For additional elements of value, HTA organization and the number/frequency of the elements of value for cancer and hepatitis $\mathrm{C}$ evaluations stratified by HTA organization in dossiers were compared using Fisher's exact test with Holm's correction, $p<0.05$.

The data analyses were performed according to the four categories mentioned in Sect. 2.3. 
Table 1 Number of dossiers used in the study stratified by Health Technology Assessment organization and disease

\begin{tabular}{lrcl}
\hline Organization & Total & Cancer & Hepatitis C \\
\hline AETSA & 12 & 5 & $7^{\mathrm{a}}$ \\
AIFA & 8 & 8 & 0 \\
CADTH/pCODR & 28 & 21 & 7 \\
C2H & 7 & 2 & 5 \\
HAS & 29 & 22 & 7 \\
IQWiG & 29 & 23 & 6 \\
NICE & 25 & 23 & 2 \\
PBAC & 51 & 43 & $8^{\mathrm{b}}$ \\
Total & 189 & 147 & 42 \\
\hline
\end{tabular}

a'Daklinza, Exviera, Harvoni, Holkira Pak, Olysio, Sovaldi, Viekirax are evaluated in one dossier for AETSA

${ }^{\mathrm{b}}$ Daklinza and Sunvepra are evaluated in one dossier for PBAC

AETSA Agencia de Evaluación de Tecnologías Sanitarias de Andalucía, AIFA Agenzia Italiana del Farmaco, $C 2 H$ Center for Outcomes Research and Economic Evaluation for Health, CADTH Canadian Agency for Drugs and Technologies in Health, HAS Haute Autorité de Santé, $I Q W i G$ Institute for Quality and Efficiency in Healthcare, NICE National Institute for Health and Care Excellence, PBAC Pharmaceutical Benefits Advisory Committee, $p C O D R$ pan-Canadian Oncology Drug Review

\section{Results}

\subsection{Dossiers Used in This Study}

A total of 189 dossiers (original, resubmission, extension of indication and unknown) were included in the study (Table 1). Over a span of 7 years, 147 cancer-related and 42 hepatitis C-related dossiers were found in the target HTA organizations up to August 2019.

PBAC had the highest number of dossiers $(n=51)$, while $\mathrm{C} 2 \mathrm{H}$ had the lowest $(n=7)$. This is due to the number of resubmissions and extensions of indication that vary by country. Resubmissions and extensions of indication account for $45 \%(n=23)$ and $44 \%(n=22)$, respectively, of total PBAC dossiers. More than $50 \%$ of dossiers by AIFA, CADTH/pCODR, HAS, IQWiG and NICE were extensions of indication for cancer drugs. Also, no dossiers for hepatitis C drugs were presented by AIFA.

Being a relatively new HTA organization (since 2018), the $\mathrm{C} 2 \mathrm{H}$ evaluates multiple diseases in one assessment for a drug, unlike the other HTA organizations (e.g. a single assessment was done for the use of Opdivo in melanoma, lung cancer and renal cancer). Also, two drugs-Ibrance and Keytruda-were not included by the $\mathrm{C} 2 \mathrm{H}$, as the HTA results and details of these drugs were not publicly available.

Moreover, the gap between the dates of marketing authorizations approved by the European Medicines Agency (EMA) and decision dates by HTA organizations in European Union countries is shown in Supplemental Table S5 (see ESM).

\subsection{Economic Evaluation Types}

The economic evaluations considered by HTA organizations, stratified by disease, are shown in Fig. 1. Irrespective of disease, all dossiers by NICE, CADTH/pCODR and $\mathrm{C} 2 \mathrm{H}$ used strictly ICER with QALY.

For IQWIG, all cancer-related dossiers were evaluated using cost-only analysis. On the other hand, only half of the hepatitis C-related dossiers were evaluated using cost-only analysis and the other half had no mention of any economic evaluations. For HAS, about $55 \%$ of dossiers showed costeffectiveness analysis using ICER with QALY while the remaining dossiers did not present any economic analysis.

For PBAC, about $85 \%$ of cancer-related dossiers presented cost-effectiveness analysis using ICER with QALY while the remaining were either cost-only analysis or no economic analysis at all. For about $75 \%$ of the hepatitis C-related dossiers, cost effectiveness was evaluated using ICER with QALY, while for the remaining, no economic analysis was presented.

AETSA and AIFA did not mention any economic evaluations in their dossiers.

\subsection{Clinical Uncertainties and Issues}

The overview of uncertainties and issues reported in cancer dossiers across HTA organizations is presented in Fig. 2. For cancer, the highest number of uncertainties observed in all HTA organizations were related to clinical benefit of the drug under consideration, with the percentage ranging from $27.3 \%(n=6)$ for HAS to $73.9 \%(n=17)$ for NICE. The second most common uncertainty reported in cancer dossiers pertained to comparators (percentages reported for uncertainties ranged from $13.0 \%$ [ $n=3$ ] for NICE to $26.1 \%$ $[n=6]$ for IQWiG). PBAC was the only HTA organization in which cancer dossiers reported uncertainties pertaining to the safety of drugs under consideration $(n=8 ; 18.6 \%$ of the submitted dossiers). Study design was reported as an uncertainty in three (13.6\%) cancer dossiers by HAS and one $(12.5 \%)$ cancer dossier by AIFA. Relatively few uncertainties were seen across all organizations for the remainder of the factors including population and sample. No data was found for $\mathrm{C} 2 \mathrm{H}$. Regarding the issues reported, a lot of variations were observed across all HTA organizations; for example, AETSA and $\mathrm{C} 2 \mathrm{H}$ reported no study-design-related issues while AIFA reported issues in the study design for $50.0 \%(n=4)$ of their submissions.

The overview of uncertainties and issues reported in hepatitis C dossiers across HTA organizations is presented 


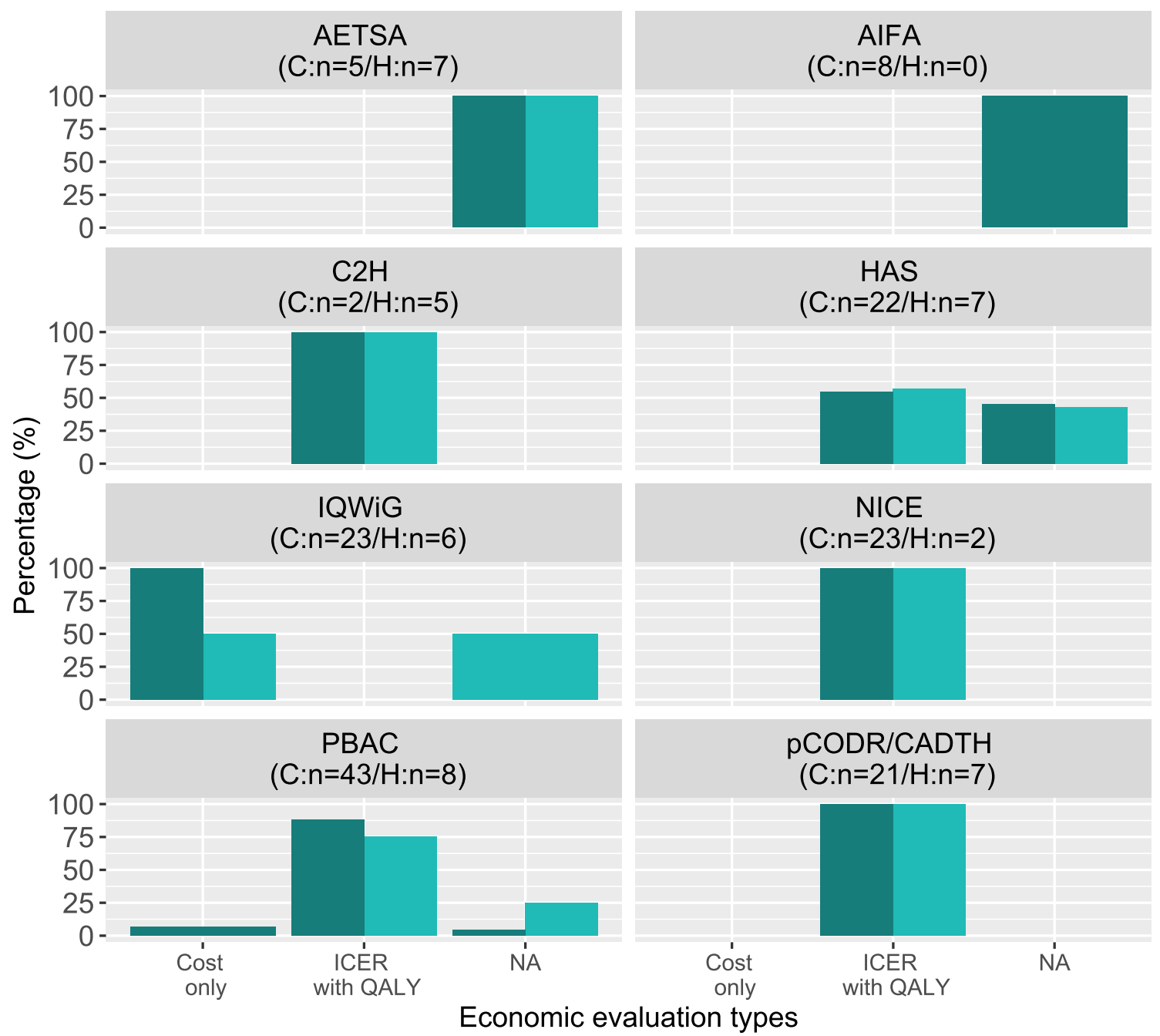

Legend Cancer Hepatitis C

$\mathrm{C}=$ Number of cancer dossiers, $\mathrm{H}=$ Number of hepatitis $\mathrm{C}$ dossiers

NA:Not available

Fig. 1 Percentages of economic evaluation types used by selected organizations and diseases. Economic evaluation types consisted of cost only, incremental cost-effectiveness ratio with quality-adjusted life-year and not available. The percentages were reported by selected organizations and diseases (cancer and hepatitis C). AETSA Agencia de Evaluación de Tecnologías Sanitarias de Andalucía, AIFA Agenzia Italiana del Farmaco, $\mathrm{C} 2 \mathrm{H}$ Center for Outcomes Research and Eco-

in Fig. 3. For hepatitis $\mathrm{C}$, similar trends in uncertainties and issues were observed compared to cancer dossiers. The most common uncertainty reported was clinical benefit and a lot of variations were observed in the reported issues. However, due to the small sample size of hepatitis $\mathrm{C}$ dossiers included in this study, any inference from the present results must be made with caution. nomic Evaluation for Health, $C A D T H$ Canadian Agency for Drugs and Technologies in Health, HAS Haute Autorité de Santé, IQWiG Institute for Quality and Efficiency in Healthcare, NICE National Institute for Health and Care Excellence, $P B A C$ Pharmaceutical Benefits Advisory Committee, $p C O D R$ pan-Canadian Oncology Drug Review

\subsection{Consideration Factors Reported by Each HTA Organization}

The consideration factors analysed across the target HTA organizations are presented in Fig. 4. Overall, the nonavailability of hepatitis $\mathrm{C}$ dossiers by AIFA; very limited information publicly released by AETSA, AIFA and $\mathrm{C} 2 \mathrm{H}$; and the low number of hepatitis $\mathrm{C}$ dossiers by NICE made 


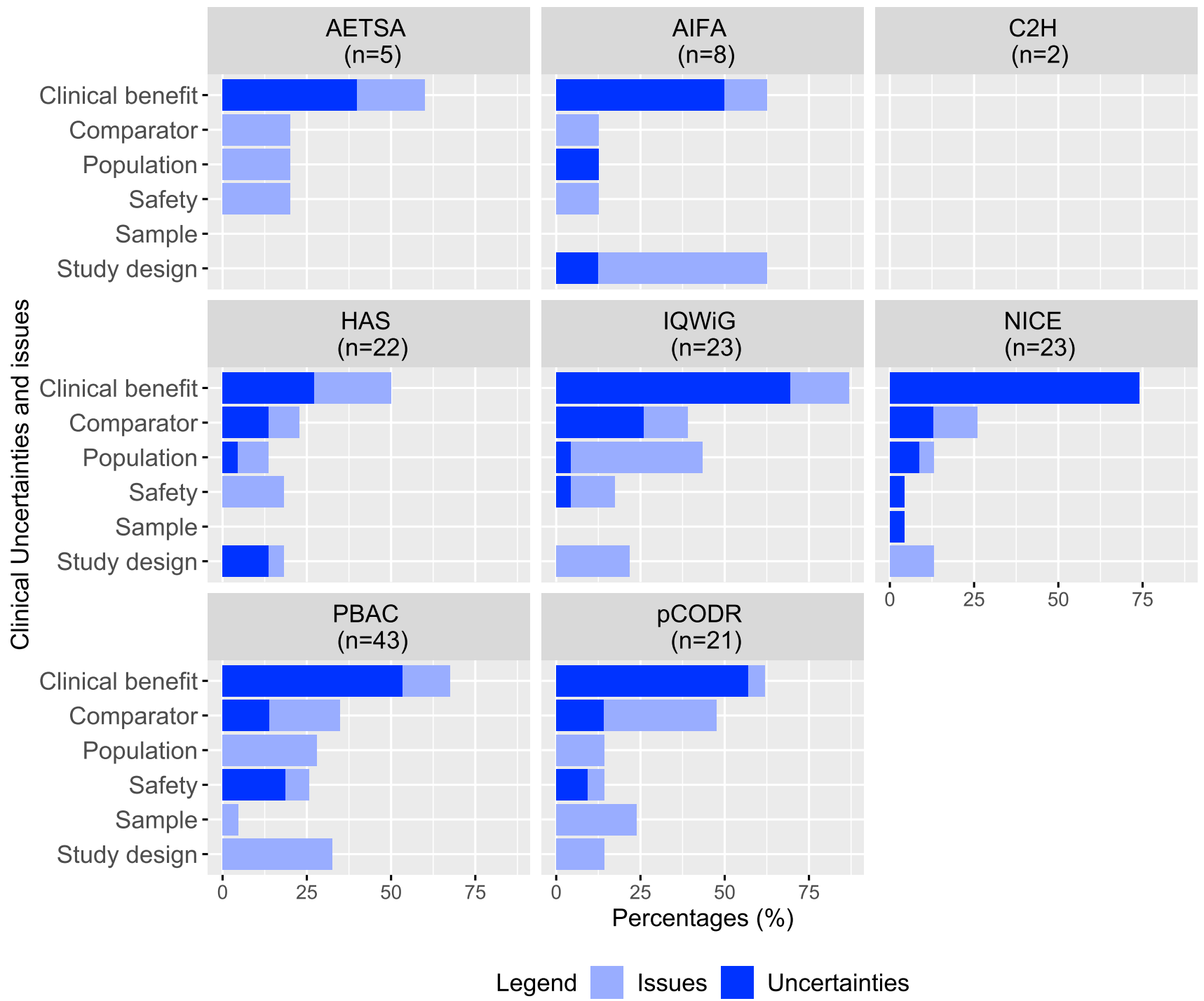

Fig. 2 Percentages of cancer clinical uncertainties and issues mentioned by Health Technology Assessment (HTA) organization. Uncertainties are defined as unclear or insufficient clinical evidences that hindered the ability to obtain a solid judgement of the assessed technology reported by the HTA organizations. Issues are defined as clinical factors that were expressed as being incorrect or problematic by the HTA organizations. AETSA Agencia de Evaluación de

it more difficult to observe trends for consideration factors across HTA organizations.

\subsubsection{Disease Considerations}

Disease severity was reported by four organizations-HAS, NICE, PBAC, CADTH/pCODR. CADTH did not mention disease factors for hepatitis C while AETSA, AIFA and IQWiG did not mention disease factors at all. National priority was observed for hepatitis $\mathrm{C}$ at the highest rate $(n=5$; $62.5 \%$ ) by PBAC. NICE reported end-of-life for cancer at
Tecnologías Sanitarias de Andalucía, AIFA Agenzia Italiana del Farmaco, $\mathrm{C} 2 \mathrm{H}$ Center for Outcomes Research and Economic Evaluation for Health, CADTH Canadian Agency for Drugs and Technologies in Health, HAS Haute Autorité de Santé, IQWiG Institute for Quality and Efficiency in Healthcare, NICE National Institute for Health and Care Excellence, PBAC Pharmaceutical Benefits Advisory Committee, $p C O D R$ pan-Canadian Oncology Drug Review

the highest rate $(n=20 ; 87.0 \%)$ of all organizations. The rare/orphan status disease factor in cancer was only presented by NICE, but at a low rate $(n=1 ; 4.3 \%)$.

\subsubsection{Population Considerations}

Amongst population factors, stakeholder persuasion was the most common factor reported across HTA organizations with a proportion of $75 \%$ or over. IQWiG and CADTH/ pCODR mentioned stakeholder persuasion for $100 \%$ of the dossiers for both cancer and hepatitis C. However, HAS, 


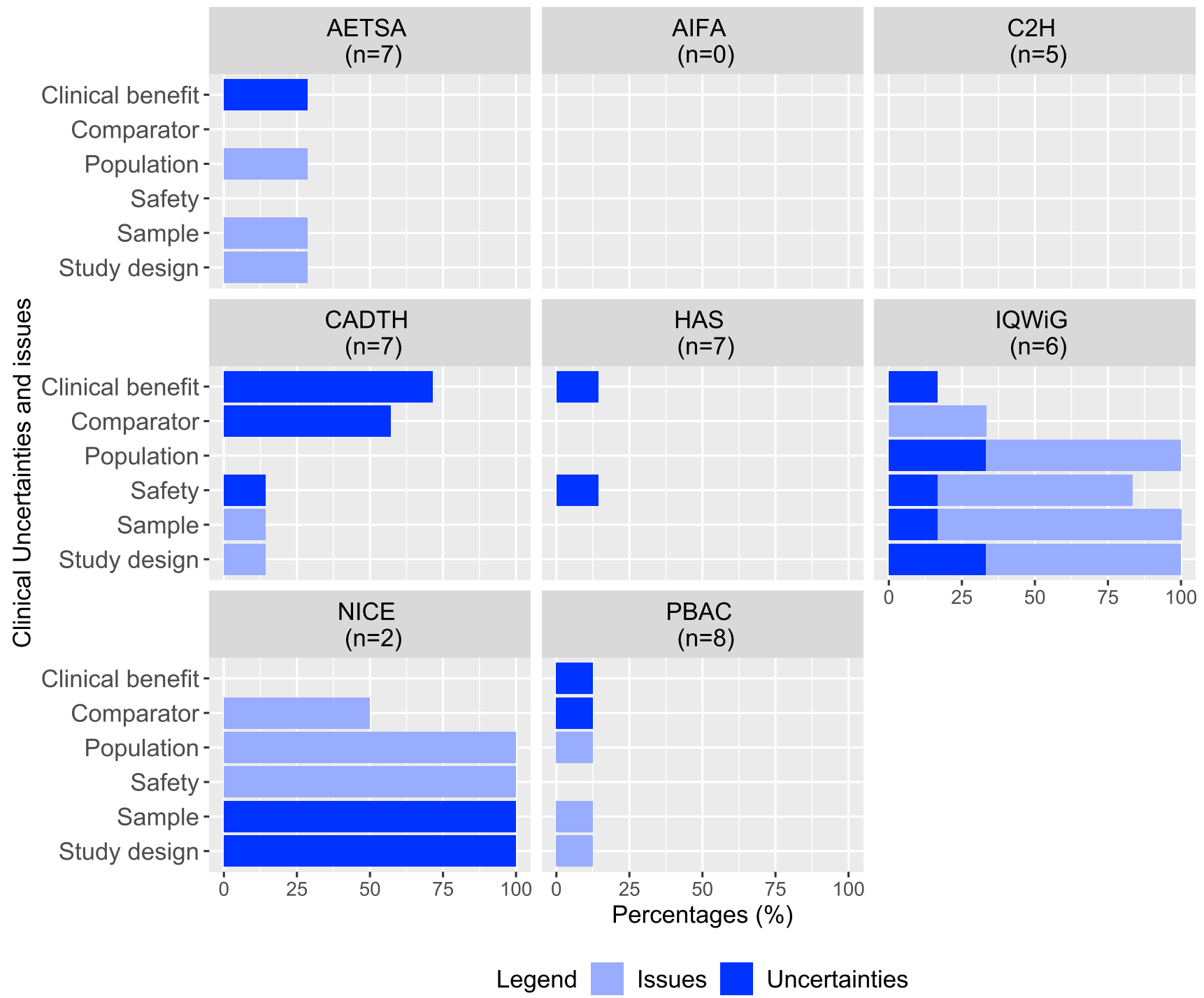

Fig. 3 Percentages of hepatitis $\mathrm{C}$ clinical uncertainties and issues mentioned by Health Technology Assessment (HTA) organization. Uncertainties are defined as unclear or insufficient clinical evidences that hindered the ability to obtain a solid judgement of the assessed technology reported by the HTA organizations. Issues are defined as clinical factors that were expressed as being incorrect or problematic by the HTA organizations. AETSA Agencia de Evaluación de

AETSA, AIFA either included it at a low rate or did not mentioned it at all. NICE noted disadvantaged population factor for both hepatitis $\mathrm{C}$ drugs evaluated.

\subsubsection{Treatment Considerations}

NICE was the only organization that reported nearly all factors related to treatment for both cancer and hepatitis $\mathrm{C}$ evaluations. NICE was also the only HTA organization to mention the complex pathways of treatment in cancer, but at a low rate $(n=2 ; 8.7 \%)$. The treatment factor observed
Tecnologías Sanitarias de Andalucía, AIFA Agenzia Italiana del Farmaco, $\mathrm{C} 2 \mathrm{H}$ Center for Outcomes Research and Economic Evaluation for Health, CADTH Canadian Agency for Drugs and Technologies in Health, HAS Haute Autorité de Santé, IQWiG Institute for Quality and Efficiency in Healthcare, NICE National Institute for Health and Care Excellence, $P B A C$ Pharmaceutical Benefits Advisory Committee, $p C O D R$ pan-Canadian Oncology Drug Review

in the highest proportion for both diseases across multiple HTA organizations was unmet needs. Six out of eight and five out of eight organizations mentioned unmet needs for cancer and hepatitis $\mathrm{C}$, respectively, at a high rate ranging from $54.5 \%$ (HAS, $n=12$ ) to $100 \%$ (AIFA, $n=8$ ). Another factor, manageable or non-significant adverse events (AEs), in cancer was also noted by six out of eight organizations, at rates ranging from $4.5 \%$ (HAS, $n=1$ ) to $78.3 \%$ (IQWiG, $n=18$ ). AIFA reported innovation and unmet needs for all cancer dossiers evaluated. 


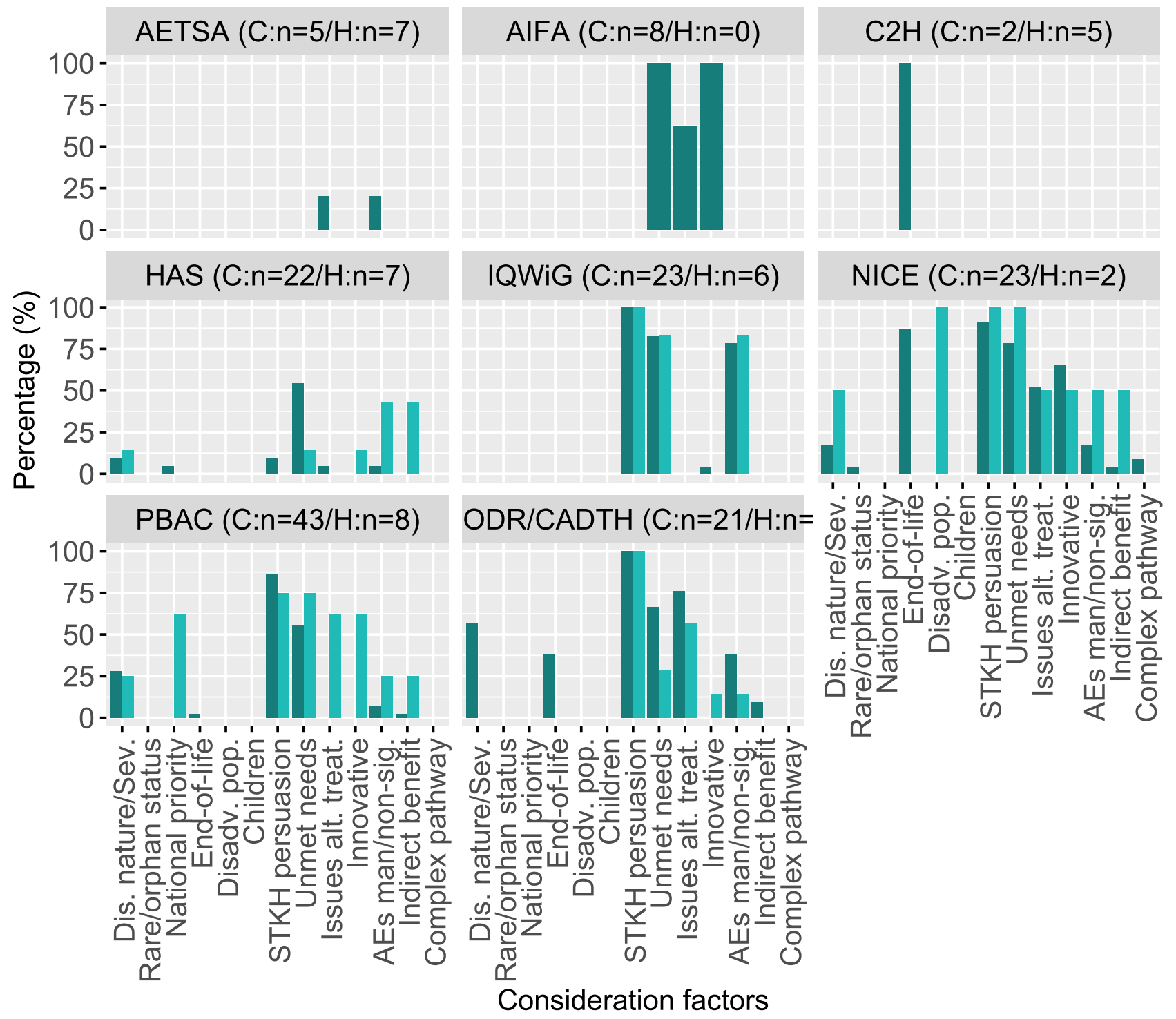

\section{Legend Cancer Hepatitis C}

C: Number of cancer dossiers, $\mathrm{H}$ : Number of hepatitis $\mathrm{C}$ dossiers

Dis. nature/Sev.: Disease nature/Severity, Disadv. pop.: Disadvantaged population, STKH persuasion: Stakehold Issues alt. treat.: Issues around alternative treatment, AEs man/non-sig.: Manageable/non-significant AEs

Fig. 4 Consideration factors across Health Technology Assessment (HTA) organizations stratified by diseases (cancer and hepatitis C). Evaluated consideration factors were categorized as follows: disease considerations (Disease nature affecting the patient/Severity of disease, Rarity, orphan status, National priority and End-of-life), population considerations (Stakeholder persuasion, Disadvantaged population and Children) and treatment considerations (Unmet needs, Issues around current treatment alternative, Complex pathway-no best practice or advance, Innovation, Indirect benefit from the treatment and Adverse events manageable/non-significant). AEs man/ non-sig. Manageable/nonsignificant adverse events, AETSA Agencia de Evaluación de Tecnologías Sanitarias de Andalucía, AIFA Agenzia Italiana del Farmaco, $C 2 \mathrm{H}$ Center for Outcomes Research and Economic Evaluation for Health, CADTH Canadian Agency for Drugs and Technologies in Health, Dis. Nature/Sev. Disease nature/Severity, Disadv. Pop. Disadvantaged population, HAS Haute Autorité de Santé, $I Q W i G$ Institute for Quality and Efficiency in Healthcare, Issues alt. treat. Issues around alternative treatment, NICE National Institute for Health and Care Excellence, PBAC Pharmaceutical Benefits Advisory Committee, $p C O D R$ pan-Canadian Oncology Drug Review, STKH persuasion Stakeholder persuasion 


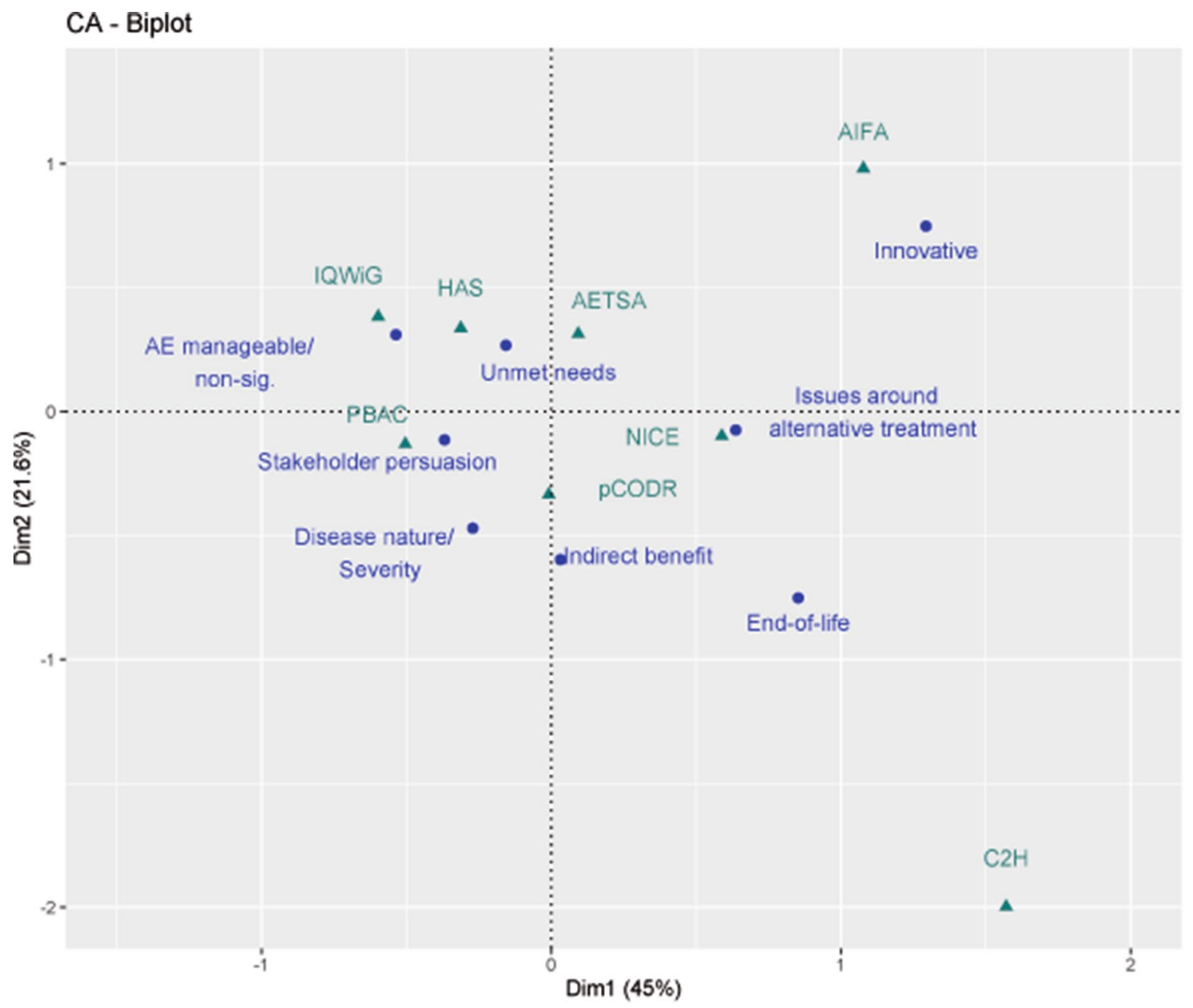

Fig. 5 Correspondence analysis for cancer drug evaluation consideration factors. Test of independence showed statistically significant association between Health Technology Assessment organizations and consideration factors $\left(\chi^{2}=234.62, p\right.$-value $\left.<0.0001\right)$. A variance of $66.6 \%$ (horizontal axis $45.0 \%$ and vertical axis $21.6 \%$ ) was accounted for by the two-dimensional representations of the first two eigenvalues. As an example, National Institute for Health and Care Excellence (NICE) was in favour of additional factors such as alterna-

The correspondence analysis illustrating the relative association between HTA organizations and the factors considered for evaluating cancer dossiers is presented in Fig. 5. Test of independence showed a statistically significant association between HTA organizations and consideration factors $\left(\chi^{2}=234.62, p<0.0001\right)$. There was a $66.6 \%$ variance was accounted by the two-dimensional representations of the first two eigenvalues.

HAS, PBAC, pCODR and NICE mentioned the maximum number of factors for their drug evaluations. The most common attributes observed across these organizations were unmet needs, stakeholder persuasion, disease nature affecting the patient and indirect benefit. NICE was distinguished from other organizations for also noting additional factors such as issues around alternative tive treatment and end-of-life and innovation, which in their plots are close together. AETSA Agencia de Evaluación de Tecnologías Sanitarias de Andalucía, AIFA Agenzia Italiana del Farmaco, $C 2 H$ Center for Outcomes Research and Economic Evaluation for Health, HAS Haute Autorité de Santé, IQWiG Institute for Quality and Efficiency in Healthcare, NICE National Institute for Health and Care Excellence, PBAC Pharmaceutical Benefits Advisory Committee, $p C O D R$ pan-Canadian Oncology Drug Review

treatment and end-of-life. IQWiG, on the other hand, was uniquely associated with manageable or non-significant AEs. AETSA, AIFA and $\mathrm{C} 2 \mathrm{H}$ reported fewer factors while evaluating drugs and in addition to their small sample size of dossiers, no confident remarks could be made.

\subsection{Additional Elements of Value Suggested by ISPOR Special Task Force}

The additional elements of value for healthcare suggested by the ISPOR Special Task Force are presented in Fig. 6. PBAC was found to be the only HTA organization currently mentioning the use of most of the additional elements suggested by ISPOR in its dossiers. The most common additional elements mentioned by PBAC were adherence-improving 


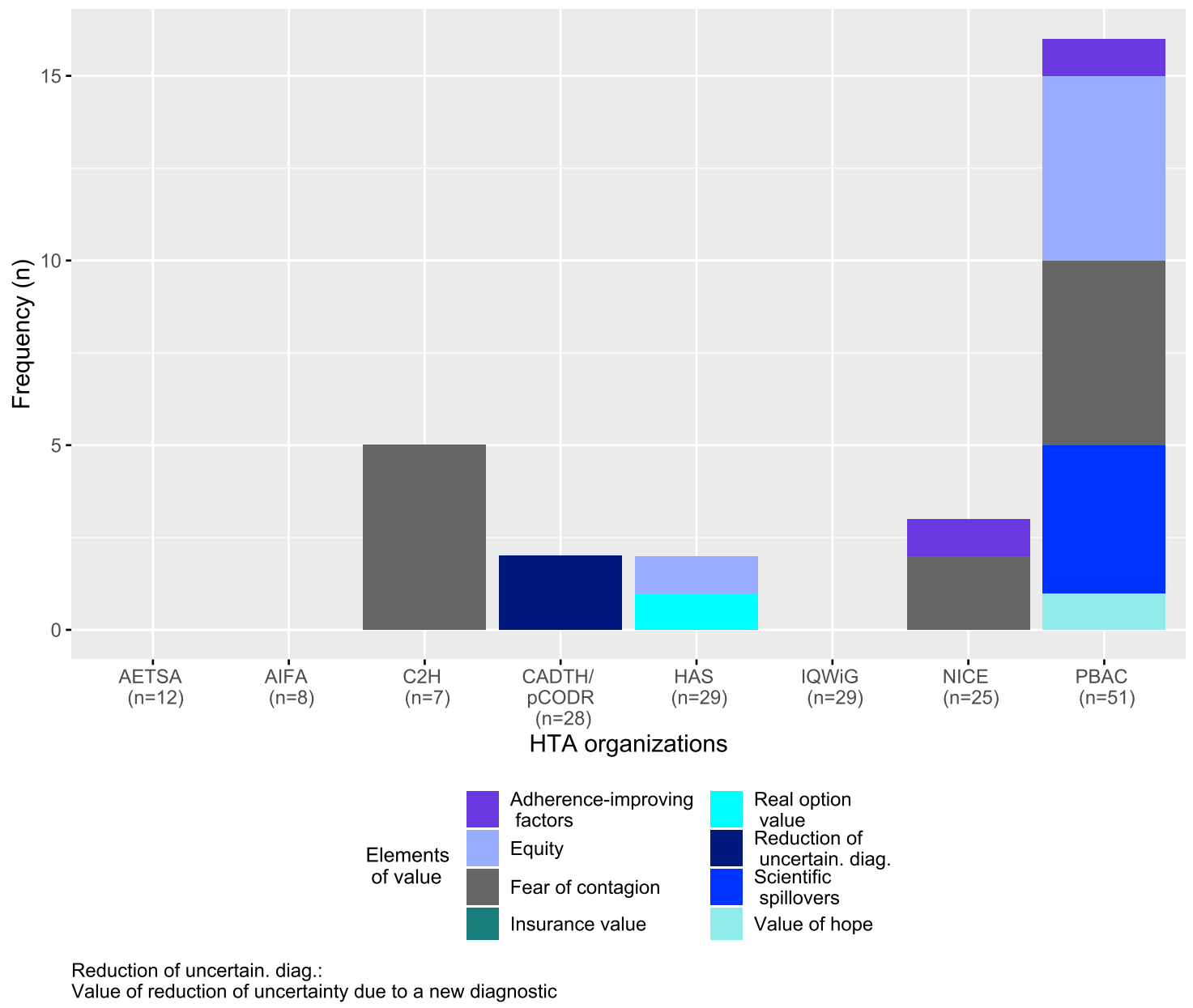

Fig. 6 Number/frequency of considered additional elements of value for cancer and hepatitis $\mathrm{C}$ evaluations stratified by Health Technology Assessment (HTA) organization. The number in the parentheses under each organization's name describes the number of dossiers. AETSA Agencia de Evaluación de Tecnologías Sanitarias de Andalucía, AIFA Agenzia Italiana del Farmaco, $C 2 H$ Center for Outcomes Research and Economic Evaluation for Health, CADTH Canadian

factors, fear of contagion, equity and scientific spillover. HAS, NICE, CADTH/pCODR and $\mathrm{C} 2 \mathrm{H}$ were found to be using one or two additional value elements while AETSA, AIFA and IQWiG were using none. The Fisher's test result can be found in Supplemental Table S6 (see ESM). The table shows the significant difference between IQWiG versus $\mathrm{C} 2 \mathrm{H}$ and versus PBAC $(p=0.0016$ and $p=0.0087$, respectively, $p<0.05)$ and HAS versus $\mathrm{C} 2 \mathrm{H}(p=0.0272, p<0.05)$.

\section{Discussion}

With increasing economic burden in the healthcare sector [1], the role of HTA organizations becomes highly important for providing reimbursement relief [26]. Furthermore,
Agency for Drugs and Technologies in Health, HAS Haute Autorité de Santé, $I Q W i G$ Institute for Quality and Efficiency in Healthcare, NICE National Institute for Health and Care Excellence, PBAC Pharmaceutical Benefits Advisory Committee, $p C O D R$ pan-Canadian Oncology Drug Review, Reduction of uncert. diag value of reduction of uncertainty due to a new diagnostic

the increasing contribution of HTA organizations in deciding the cost effectiveness of new interventions makes them even more significant [6]. There has been a high variability observed in the recommendation trends of HTA organizations across countries for the same intervention [10,11, 27]. Hence, it becomes important for stakeholders, such as healthcare professionals, patients and pharmaceutical companies, to understand the methodologies and factors considered by HTA organizations for decision making.

The present study reviewed four types of factors for the decision making across eight HTA organizations-economic evaluation types, clinical uncertainties/issues, consideration factors and additional value elements.

It is important to note that the economic evaluations across HTA organizations are utilized for different stages of 
the HTA process. Cost-effectiveness analysis using ICER with QALY was evaluated by CADTH/pCODR, NICE and PBAC for the purpose of decision making around reimbursements. IQWiG also presented economic evaluations for the decision making of reimbursements using budget impact analysis for all cancer and 50\% of the hepatitis C dossiers. On the other hand, HAS and $\mathrm{C} 2 \mathrm{H}$ utilized cost effectiveness using ICER with QALY for price adjustments rather than for reimbursement decision making [28, 29]. Note that Japan only formally introduced HTA and, more specifically, cost-effectiveness analysis in April 2019, after initiating a pilot programme that started in April 2016 [30].

The clinical factors were evaluated in two areas-uncertainties and issues. We observed more uncertainties (approximately 80\%) than issues (approximately 20\%) for clinical benefits across all HTA organizations. The uncertainties and issues reported across organizations were variable and were broadly related to comparators, population, safety and sample size. Since these factors also indirectly influence the clinical benefit of an intervention, any uncertainties and issues pertaining to them would have automatically led to the higher rate of uncertainties and issues for clinical benefit. These findings were in line with the results of a study conducted by Nicod that observed high rates of uncertainties for clinical benefit and study design for NICE [13]. It is expected that clinical uncertainties and issues have an impact on the HTA decisions regarding reimbursements. In fact, in Japan, cost-effectiveness analysis of any intervention is only conducted if it has met all the criteria of clinical evidence. This might also be the reason for the absence of any clinical uncertainties by $\mathrm{C} 2 \mathrm{H}$ since their main objective is to assess cost-effectiveness analyses [28].

Amongst consideration factors, stakeholder persuasion and unmet needs were the most common factors for decision making across all HTA organizations. Stakeholder persuasion is usually voiced by patients and/or healthcare professionals and HTA bodies can decide whether to accept it or not. Unmet needs are related to clinical efficiency, safety and patient demand. Given that the interventions included in this study were not children-specific or for a rare disease, it was expected that children and rare/orphan status factors would not be regarded in most HTA dossiers. For hepatitis $\mathrm{C}$ drug assessments, NICE discussed certain minority ethnic groups as disadvantaged populations. In addition, end-of-life was also mentioned by NICE given that they do give special consideration to this factor, specifically for cancer dossiers [31]. However, it is difficult to confidently conclude that disadvantaged population and end-of-life factors are unique to NICE, recognizing that other HTA organizations might equally be considering these factors, but they might simply not be stated in the HTA dossiers. As seen in Nicod [13], unmet needs is a highly observed factor. However, there are some differences in results as this study evaluates cancer and hepatitis $\mathrm{C}$ drugs rather than orphan drugs. For example, the rates for rare/orphan status and national priority would be more significant for orphan drugs.

For potential elements of value in healthcare, the ISPOR Special Task Force has suggested certain factors to broaden the considerations for decision making. Most of the suggested elements, such as fear of contagion (5 of 12), equity (5 of 6) and scientific spillovers ( 4 of 4 ) were being reported by PBAC only. PBAC mentions fear of contagion for hepatitis $\mathrm{C}$ treatments that have the potential to reduce disease transmission. It also considers equity for the wellbeing of the general public as the awareness of options for hepatitis $\mathrm{C}$ treatment increases. Scientific spillover was mentioned pertaining to the probable creation of new similar treatments for hepatitis $\mathrm{C}$ in the future.

In this study, the correspondence analysis was performed to display the relationship between HTA organization and consideration factor as a useful tool to clarify the relationship between categorical variables. However, correspondence analysis is not suitable for a hypothesis test. In the future, statistical analysis to reveal the relationship based on a hypothesis test is needed for better decision making.

There are other limitations of this research. This research could not determine included or absent factors because of missing information about the existence of the factors in HTA dossiers. Therefore, the factors were raised up as potential factors to be included in each HTA dossier. There is a reliance on Google translation of non-English dossiers, reflecting possible language misinterpretation. However, Ethan et al. (2012) showed that Google translation provides a certain degree of accuracy, with some caution required [32]. Due to the low sample size of hepatitis $C$ dossiers, caution is recommended when interpreting the results. Also, although HTA organizations in countries that have similar health expenditure were selected to remove the heterogeneities of different approvals and reimbursements for the drugs investigated, and to correspond the findings from the documents in selected HTA organizations, a lot of uncertainties remained in our results. These uncertainties are associated with the public accessibility or transparency of the HTA process and result. Akehurst et al. [9] reported that there was a lack of transparency in the long duration process for some products. Epstein and Espín [33] compared the process and results of European HTA agencies and reported most organizations such as HAS, NICE and IQWIG are far from perfect in terms of transparency [9, 33].

This study employed a new approach, which compared the HTA process for drugs of interest across developed countries including Japan. This is because a pilot HTA programme was introduced in Japan in April 2016. 


\section{Conclusion}

This study has provided a view into the additional factors that could be leveraged in broadening the knowledge of reimbursement processes. Although clinical factors play a predominant role in the decision to reimburse medicines, NICE and PBAC were found to be the HTA organizations with the most comprehensive list of additional criteria. Furthermore, CADTH/pCODR, HAS and IQWiG also showed a direction for decision making that extends beyond clinical evidence. If the decision-making process of HTA were clearly outlined with more public accessibility or transparency into the considered factors, there would be more transparency in HTA systems, leading to better understanding amongst stakeholders about decision making.

Acknowledgements This study includes the research content that A.Y. conducted during his course at the International University of Health and Welfare. Raf De Moor, Hiroyuki Matsuda and Ryo Mitsuo (employees of IQVIA Solutions Japan K.K.) contributed to searching and checking the HTA dossiers.

\section{Declarations}

Funding Pfizer Japan Inc. was the only direct sponsor of this study and fees were paid to IQVIA Solutions Japan K.K. A.Y. and N.Y. are employees of Pfizer Japan Inc. and substantially contributed to the conception, design, analysis of data and the subsequent drafting of this work.

Conflict of interest The authors declare the following potential conflicts of interest with respect to the research, authorship and/or publication of this article: A.Y. and N.Y. are full-time employees of Pfizer Japan Inc. S.D. and C.F. are full-time employees of IQVIA Solutions Japan K.K. S.I. declared no conflicts of interest associated with this manuscript.

Ethics approval Not applicable.

Consent to participate Not applicable.

Consent for publication Not applicable.

Availability of data and material The data set used for the analysis was gathered from various public domains (i.e. the agencies' websites) and are hence publicly available, but are also available from the corresponding author upon reasonable request. Standard statistical function was used in $\mathrm{R}$ and no additional software code was developed.

Code availability The datasets used and/or analysed during the current study are available from the corresponding author on reasonable request.

Author Contributions All authors contributed to the study design, analysis, interpretation, writing manuscript and review. C.F., R.D.M., H.M. and R.M. contributed to the data collection.

Open Access This article is licensed under a Creative Commons Attribution-NonCommercial 4.0 International License, which permits any non-commercial use, sharing, adaptation, distribution and reproduction in any medium or format, as long as you give appropriate credit to the original author(s) and the source, provide a link to the Creative Commons licence, and indicate if changes were made. The images or other third party material in this article are included in the article's Creative Commons licence, unless indicated otherwise in a credit line to the material. If material is not included in the article's Creative Commons licence and your intended use is not permitted by statutory regulation or exceeds the permitted use, you will need to obtain permission directly from the copyright holder. To view a copy of this licence, visit http://creativecommons.org/licenses/by-nc/4.0/.

\section{References}

1. Xu K, Soucat A, Kutzin J. Public spending on health: a closer look at global trends. 2018.

2. Canadian Institute for Health Information. Health care cost drivers: the facts. 2011.

3. Dixit S, Sambasivan M. A review of the Australian healthcare system: a policy perspective. SAGE Open Med. 2018;6:21.

4. Stepanova M, Younossi ZM. Economic burden of hepatitis C infection. Clin Liver Dis. 2017;21(3):579-94.

5. World Health Organisation. Pricing of cancer medicines and its impacts. Geneva: WHO; 2018.

6. Drummond MF, Sculpher MJ, Claxton K, Stoddart GL, Torrance GW. Methods for the economic evaluation of health care programmes. Oxford: Oxford University Press; 2015.

7. Koçkaya G, Wertheimer A. Pharmaceutical market access in developed markets: SEEd; 2018.

8. Beletsi A, Koutrafouri V, Karampli E, Pavi E. Comparing use of health technology assessment in pharmaceutical policy among earlier and more recent adopters in the European Union. Value Health Reg Issues. 2018;16:81-91.

9. Akehurst RL, Abadie E, Renaudin N, Sarkozy F. Variation in health technology assessment and reimbursement processes in Europe. Value Health. 2017;20(1):67-76.

10. Allen N, Walker SR, Liberti L, Salek S. Health technology assessment (HTA) case studies: Factors influencing divergent HTA reimbursement recommendations in Australia, Canada, England, and Scotland. Value Health. 2017;20(3):320-8.

11. Cameron D, Ubels J, Norström F. On what basis are medical cost-effectiveness thresholds set? Clashing opinions and an absence of data: a systematic review. Glob Health Action. 2018;11(1):1447828.

12. Dakin H, Devlin N, Feng Y, Rice N, O'Neill P, Parkin D. The influence of cost-effectiveness and other factors on nice decisions. Health Econ. 2015;24(10):1256-71.

13. Nicod E. Why do health technology assessment coverage recommendations for the same drugs differ across settings? Applying a mixed methods framework to systematically compare orphan drug decisions in four European countries. Eur J Health Econ. 2017;18(6):715-30.

14. Rawlins M, Barnett D, Stevens A. Pharmacoeconomics: NICE's approach to decision-making. Br J Clin Pharmacol. 2010;70(3):346-9.

15. Lakdawalla D, Doshi J, Garrison L, Phelps C, Basu A, Danzon $P$. Defining elements of value in health care- a health economics approach: an ISPOR special task force report. Value Health. 2018;21(2):131-9.

16. Enrique S-V, Hans M, Jose-Manuel RB, Sheryl S. Spain-pharmaceutical global health technology assessment road map 2009 [cited $2020 \mathrm{March}$ ]; Available from: https://tools.ispor.org/htaro admaps/Spain.asp 
17. Instituto Nacional de Estadistica. Population Figures 1 July 2018, Migrations Statistics. First half of 2018. 2018.

18. Organisation for Economic Co-operation and Development. Health spending. [cited 2020 March]; Available from: https:// data.oecd.org/healthres/health-spending.htm

19. The World Bank. GDP per capita (current US\$). [cited 2020 March]; Available from: https://data.worldbank.org/indicator/ NY.GDP.PCAP.CD?most_recent_value_desc $=$ false

20. IQVIA. Leveraging HTA Accelerator. [cited 2020 March]; Available from: https://www.iqvia.com/landing/hta-accelerator

21. Dekkers RVG, van Engen A (Ultra-)orphan drug assessment in Poland: impact of proposed changes on HTA outcomes (Poster) ISPOR Europe 2018. Barcelona 2018.

22. Verghese N, Barrenetxea J, Bhargava Y, Agrawal S, Finkelstein E. Government pharmaceutical pricing strategies in the Asia-Pacific region: an overview. J Mark Access Health Policy. 2019;7(1):1601060.

23. Hoffman D, Franke G. Correspondence analysis: graphical representation of categorical data in marketing research. J Mark Res. 1986;23(3):213-27.

24. Lê S, Josse J, Husson F. FactoMineR: an R package for multivariate analysis. J Stat Softw. 2008;25(1):1-18.

25. Nenadic O, Greenacre M. Correspondence analysis in R, with two-and three-dimensional graphics: the ca package. J Stat Softw. 2007;20:3.
26. Health Technology Assessment International. About HTAi. [cited $2020 \mathrm{March}$ ]; Available from: https://htai.org/about-htai/

27. Patel P, Soumalevris A. PCN122-Drivers for different treatment cost estimations across HTA bodies. Value Health. 2018;21:S35.

28. Sakamoto H, Rahman M, Nomura S, Okamoto E, Koike S, Yasunaga H, et al. Japan health system review. 2018.

29. Haute Autorité de S. Pricing \& reimbursement of drugs and HTA policies in France. 2014.

30. Hasegawa M, Komoto S, Shiroiwa T, Fukuda T. Formal implementation of cost-effectiveness evaluations in Japan: a unique health technology assessment system. Value Health. 2020;23(1):43-51.

31. National Institute for Health and Care Excellence. Breast cancer (advanced or metastatic)—lapatinib: appraising life-extending, end of life treatments. London: National Institute for Health and Clinical Excellence; 2009.

32. Balk EM, Chung M, Hadar N, Patel K, Yu WW, Trikalinos TA, et al. AHRQ methods for effective health care accuracy of data extraction of non-english language trials with google translate. Rockville: Agency for Healthcare Research and Quality; 2012.

33. Epstein D, Espín J. Evaluation of new medicines in Spain and comparison with other European countries. Gac Sanit. 2020;34(2):133-40. 